\title{
Analyze Gear Failures and Identify Defects in Gear System for Vehicles Using Digital Image Processing
}

\author{
${ }^{1}$ A. Dasthagiraiah, M. Tech, ${ }^{2}$ N. V. Subba ReddyM.Tech, ${ }^{3,}$ N. Ramanamma, \\ ${ }^{4}$ K. Harshavardhan Reddy, ${ }^{5}$ V. Naresh Reddy, ${ }^{6}$ K. Rama Devi., \\ 1,2Assistant Professors in ECE Department, MRRITS,Udayagiri,SPSR Nellore(D.t). \\ 3,4,5,6 UG (B.Tech)Students in ECE Department, MRRITS,Udayagiri, SPSR Nellore(D.t).
}

\begin{abstract}
Gear defects are a major reason for poor quality and of embarrassment for manufacturers. 0 Inspection processes done on these industries are mostly manual and time consuming. To reduce error on identifying gear defects requires more automotive and accurate inspection process. Considering this lacking, this research implements a Gear Defect Recognizer which uses computer vision methodology with the combination of local thresholding to identify possible defects. The recognizer identifies the gear defects within economical cost and produces less error prone inspection system in real time. In order to generate data set, primarily the recognizer captures digital gear images by image acquisition device and converts the RGB images into binary images by restoration process and local threshold techniques. Later, the outputs of the processed image are the area of the faulty portion and compute the possible defective and non-defective gear as an output. Detection of bad quality plastic gears is critical for any manufacturing unit trying to make a mark in the market in terms of quality standard and cost. Here we explore the possibility of using image segmentation and algorithms like non-smooth surface detection algorithms to automate the process of defect detection. In these plastics we have picked industrial strength plastic gears used typically in applications like robotic arms where quality in paramount for the functioning of the device. In this paper review of various gear defects and the possible automated solutions using image processing techniques for defect detection is given.

Keywords: Defect detection, image processing, computer vision, thresholding, counting number of teeth's.
\end{abstract}

\section{Introduction}

All plastic industries aim to produce various competitive plastic products. The competition enhancement depends mainly on productivity and quality of the plastic produced by each industry. In this sector, there have been an enlarge amount of losses due to defective products. Most defects arising in the production process are still detected by human inspection. The work of inspectors is very tedious and time consuming. The identification rate is about $70 \%$. In addition, the effectiveness of visual inspection decreases quickly with fatigue. Digital image processing techniques have been increasingly applied to plastic gear samples for analyzing the product. As the technological progress is [1] happening the products are now extensively made using plastic material especially in robotics which needs to be ultra light weight and modular in nature plastic components like gears, As per industry statistics we have found that gears are made up of plastic material High-density polyethylene (HDPE) [1] which is prone to various kinds of defects when manufacturing using image processing. Therefore we suggest a fully robust system taking advantage of image processing techniques (Image segmentation, Non smooth corner detection etc) must be explored to build an economical solution to provide Total Quality Management[2] in manufacturing units which would allow an eco-system of continuous monitoring and improvement there by reducing the cost. This paper is organized into Section I includes Introduction, Section II Related work, Section III Model Presentation, Section IV Results and Section V Conclusion and future work.

\section{Types Of Defects In Plastic Gears}

Before we go further to explain the techniques involved in image processing for improving quality in plastic manufacturing unit, let us first consider and overview the types of defects of plastic products like gears. The various types of defects [2] that can be present in the plastic gears which can be detected by using image processing are:-

\section{A. Flash}

This defect refers to the excess molding material that penetrates into mold gaps like slide push-out faces, and inserts, etc. in a olten state. 


\section{B. Warping}

This defect describes the deformation which occurs when there are differences in the degree of shrinkage at different ocations within the molded component.

\section{Bubbles}

It is an air bubble like material trapped inside plastic gear as a defect during its production.

\section{Unfilled sections}

This defect occurs when injection molding does not reach certain portions of the inner side of the die before solidifying.

\section{E.Sink marks}

These are the marks or irregular patches on the surface that occur on the outer surfaces of molded components.

\section{E. Ejector marks}

Flow marks in which a pattern of the flow tracks of the molten plastic remains on the surface of the molded product.

\section{Related Work Done}

Alisha Tramline et.al [3] were able to understand how mould and die when not working properly, might give rise to the surface defects in plastic product. This paper basically discuss defects develop due to shrinkage, due to overheating and variation in temperature. To identify these kinds of defects, this paper suggests using SEM (Scanning Electron Microscope) technique. This algorithm was running on the surface as well as the crosssections of the plastic products. The main objective of this paper was to perform a systematic study of several types of internal defects that can be occasionally found in forged products and identify the defect surface prior to and after high temperature heat treatment. S. Kamaruddin et.al [4] paper presented a study in which an attempt has been made to improve the quality characteristic (shrinkage) of an injection molding product (plastic tray) made from blends plastic (75\% polypropylene (PP) and 25\% low density polyethylene (LDPE)) by optimizing the injection molding parameters using the Taguchi method. This paper had made an attempt to describe the optimization of the injection moulding process parameters for optimum shrinkage performance of a plastic tray which is made from polymer blends or polyblends. The performance of the plastic trays was evaluated in terms of its shrinkage behavior. The analysis of the results shows that the optimal combinations for low shrinkage are low melting temperature, high injection pressure, low holding pressure, long holding time and long cooling time.

Using Taguchi method for design of experiment (DOE), other significant effects such as interaction among injection molding parameters are also investigated. Bernd Scholz-Reiter et.al [5] introduced a machine vision based quality concept for the surface inspection of micro parts using these kinds of three dimensional images, called height maps. Quality control, the use of flexible and robust machine vision techniques are necessary because the exact positioning of micro-parts was not always given and has to be virtually compensated.

By obtaining high-resolution optical images with depth selectivity, it allows three- dimensional reconstructions of topologically-complex objects. This paper outlines a machine vision based quality concept for the surface inspection of micro-parts being manufactured in the Collaborative Research Center (CRC).To detect manufacturing errors on the surface, the neighborhood of each pixel is scanned and the deviation to the arithmetic mean of the pixel values are computed which correspond to neighborhood area. To detect manufacturing errors on the surface, the neighborhood of each pixel is scanned and the deviation to the arithmetic mean of the pixel values from the corresponding neighborhood area is computed.

\section{Model Presentation}

The system design of gear defect recognizer, which mentioned into this paper, is illustrated in Fig. 1. The proposed system can be a competitive model for recognizing gear defects in real world. Base on the research, the proposed system design is separated into two parts. The first part of our research processes the images to calculate the thresholding values of different gears. The second part calculates the number of gears and third part checks whether the gear is defective or non-defective.

\section{A. Processing Input Using Computer vision methodology:}

In our recognition system, the original digital (RGB) image is converted into grayscale image through noise removing and filtering techniques (restoration process). As image processing is costly, for this reason, adaptive median filter algorithm has been used as spatial filtering for minimizing time complexity and maximizing performance. After restoration processing, we calculate threshold value of grayscale image. In our 
proposed system, the most important key point is the decision tree processing in order to achieve the threshold value. As we know that there have been different types of color gear images and also different types of defects in gears, so local thresholding was used based on decision tree process. We have identified the threshold value (T) at greater than 120 and less than 60. Due to different threshold values to different pattern of faults of gears, we generalize a specific threshold value $(t)$ for all types of gears. Base on the threshold value achieved from the decision tree, grayscale image is converted into binary image using local thresholding technique.

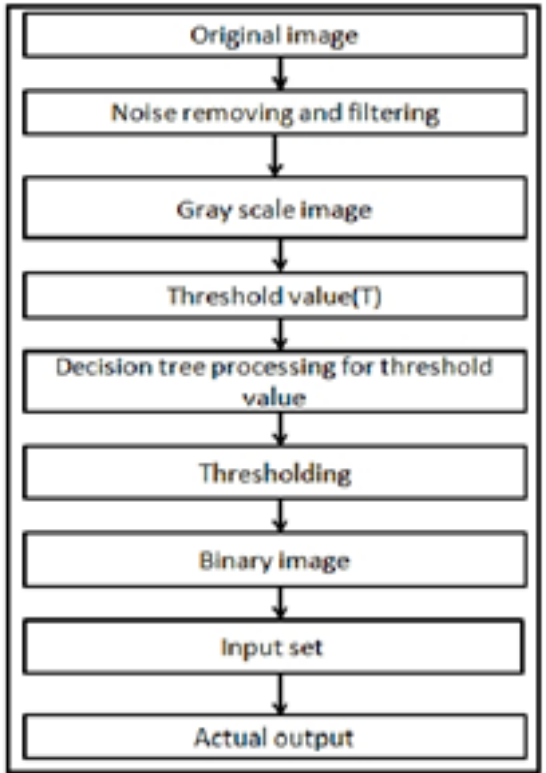

Figur 1, System Design of Textile Defect Recognizer

\section{B. Teeth counting algorithm of gear:}

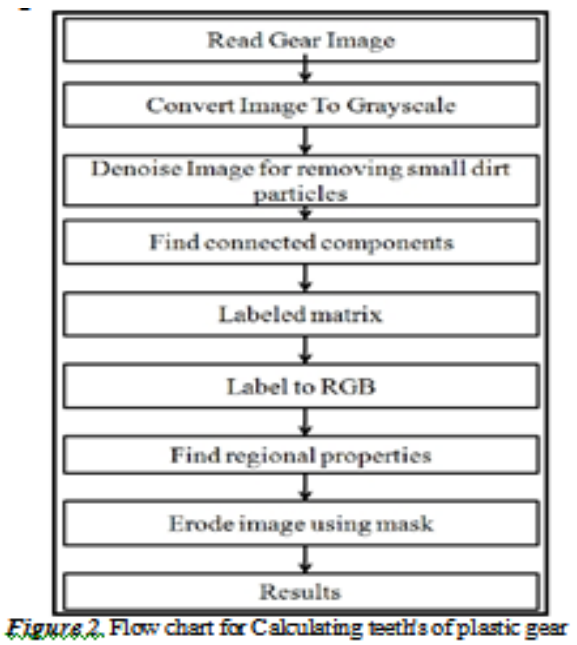

The steps for teeth calculating algorithm of plastic gear is as follows:-

1.The input image is read by using imread function.

2.The algorithm can be tested for gray scale and a color image by appropriately using functions such as is gray function.

3.Denoise the gear image for removing the small dirt particles.

4.Find the connected components of the gear image .Pixels are connected if their edges touch. This means that a 5.pair of adjoining pixels are part of the same object only if they are both on and are connected along the 6.horizontal or vertical direction..

7.Generate the labeled matrix and produce an RGB image for highlighting the gear image. Extract regional properties of the gear image. Erode image. Results. 


\section{Algorithm for purposed work}

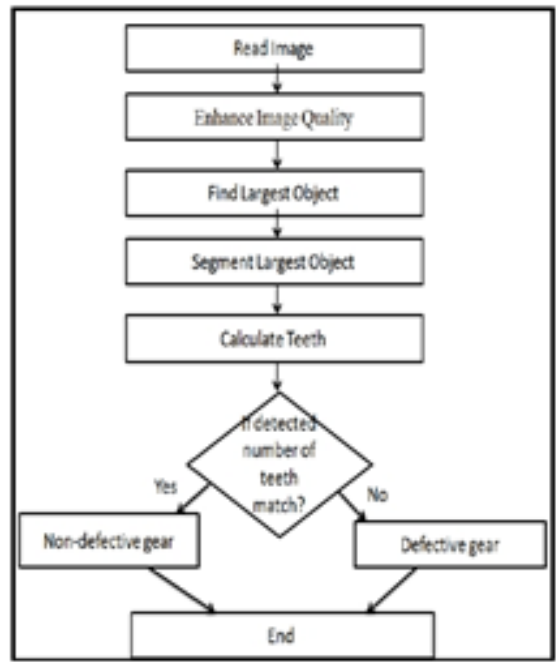

Figure 3. Flow chart of the purposed method

The proposed algorithm is mentioned as follows:-

1.The input image is read by using imread function.

2.The algorithm can be tested for gray scale by appropriately using functions.

3.Enhancement image quality by using the spatial filters which operate on pixel values.

4.Denoising -Noise is the result of errors in the image acquisition process that result in pixel values that do not reflect the true intensities of the real scene.

5.Segment largest object i.e. gear image.

6.Calculate the labels i.e. number of teeths of gear.

7.If the number of teeth match with the subscribe number then the gear is non-defective, otherwise the gear is defected.

\section{How to Analyze Gear Failures:}

When an important gear failure occurs, someone becomes responsible for analyzing the failure, determining its cause and recommending a solution. A company can select its own engineer, an outside consultant or both. If a consultant is called in, this should be done as early in the process as possible.

Though similar procedures apply to any failure analysis, the specific approach can vary depending on when and where the inspection is made, the nature of the failure and time constraints.

When and where. Ideally, the engineer conducting the analysis should inspect the failed components as soon after failure as possible. If an early inspection is not possible, someone at the site must preserve the evidence based on instructions from the analyst.

If a suitable facility for disassembling and inspecting the gearbox is not available onsite, it may be necessary to find an alternate location or bring the necessary equipment to the site.

Nature of failure. The failure conditions can determine when and how to conduct an analysis. For example, if the gears are damaged but still able to function, the company may decide to continue their operation and monitor the rate at which damage progresses. In this case, samples of the lubricant should be collected for analysis, the reservoir drained and flushed and the lubricant replaced.

If gearbox reliability is crucial to the application, the gears should be examined by magnetic particle inspection to ensure that they have no cracks. The monitoring phase will consist of periodically checking the gears for damage by visual inspection and by measuring sound and vibration.

Time constraints. In some situations, the high cost of shutting down equipment limits the time available for inspection. Such cases call for careful planning. For example, dividing tasks between two or more analysts reduces the time required.

The load capacity of the gearset will need to be calculated later. For this purpose, obtain the following geometry data, either from the gears and gear housing or their drawings:

- Number of teeth

- Outside diameter

- $\quad$ Face width

- Gear housing center distance for each gearset 
- Whole depth of teeth

- Tooth thickness (both span and top land measurement)

Specimens for laboratory tests. During the inspection, you will begin to formulate hypotheses regarding the cause of failure. With these hypotheses in mind, select specimens for laboratory testing. Take broken parts for laboratory evaluation or, if this is not possible, ensure that they will be preserved for later analysis.

Oil samples can be very helpful. But, an effective lubricant analysis depends on how well the sample represents the operating lubricant. To take samples from a gearbox drain valve, first discard stagnant oil from the valve. Then take a sample at the start, middle and end of a drain to avoid stratification. To sample from the reservoir, draw samples from the top, middle and near the bottom. Examine the oil filter and magnetic plug for wear debris and contaminants.

Samples from the oil storage drum or reservoir can uncover problems such as excessive water in the oil due to improper storage.

Have you got it all? Before leaving the site, make sure that you have everything needed (completed inspection forms, written descriptions and sketches, photos and test specimens) for completing the failure analysis.

Conduct nondestructive tests before any destructive tests. These nondestructive tests, which aid in detecting material or manufacturing defects and provide rating information, include:

- Surface hardness and roughness.

- Magnetic particle inspection.

- Acid etch inspection.

- Gear tooth accuracy inspection.

Then conduct destructive tests to evaluate material and heat treatment. These tests include:

- Microhardness survey.

- Microstructural determination using various acid etches.

- Determination of grain size.

- Determination of nonmetallic inclusions.

- SEM microscopy to study fracture surfaces.

\section{Results}

To see the qualitatively as well as quantitatively performance of the proposed algorithm, some experiments are conducted on several colored and gray scale images. The effectiveness of the approach has been justified using different images. The results are computed qualitatively (visually) as well as quantitatively using quality measures.

The figures from Figure 4 to Figure 13 are the screenshots of the proposed work which shows the different images which consists of original images and output gear images. 


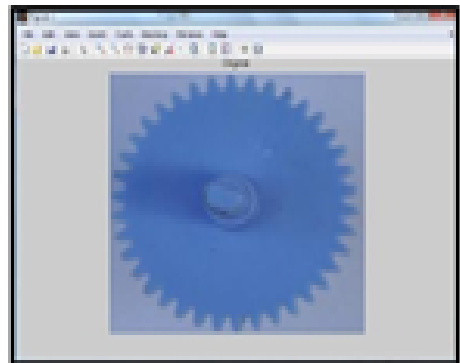

Figure 4. Original gear image

This is the RGB image of the original platic gear which is used as an irput.

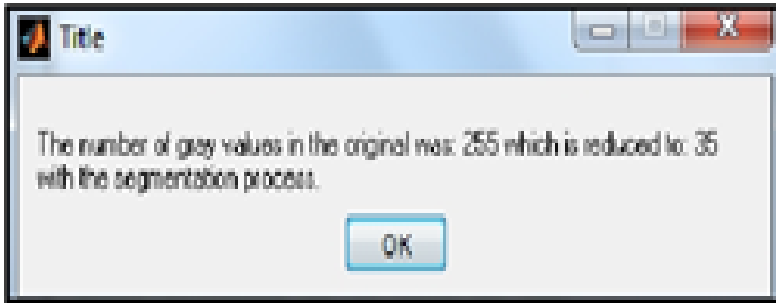

Figure 5 Segmentationproces

Here the number of the gray walua in the value was 255 which have been reduced to 35 with segnentation proces

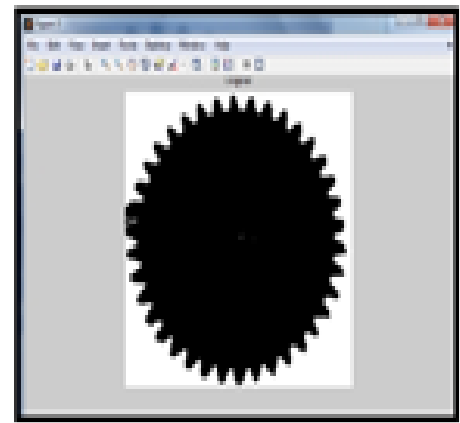

Figure 6.Gryacale inage

The gear images have ben convented into blak by using the complement oode for inreasing the visibilty.

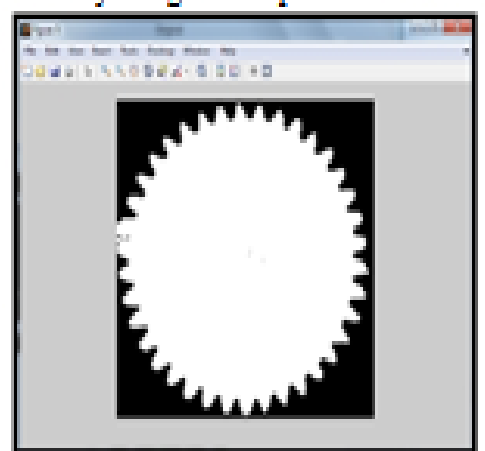

Figure 7Binaryimage

The above figure image shows the binary image for increasing the visibility with respect to the surface. 


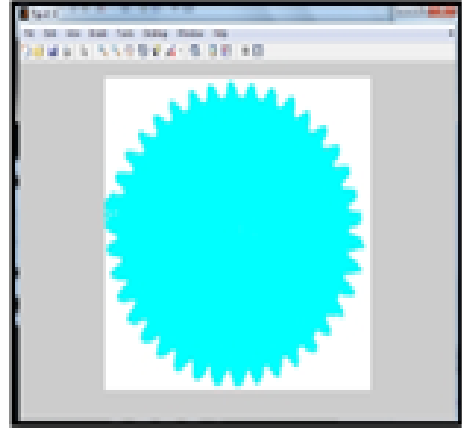

Figure \&RCB ingge

This is the colored image of a gear for highlighting the gear part.

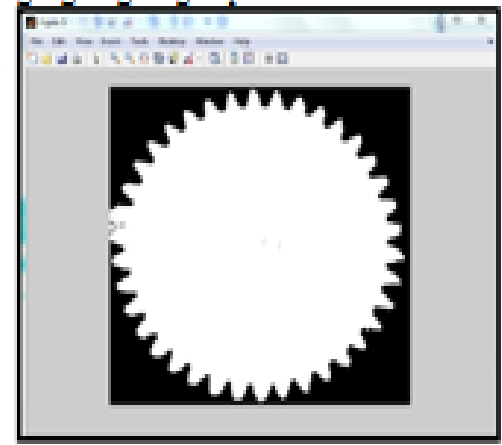

Figure 9. Input ingge

This is the birarizad image of the gear passing through the Teeth counting algorith.

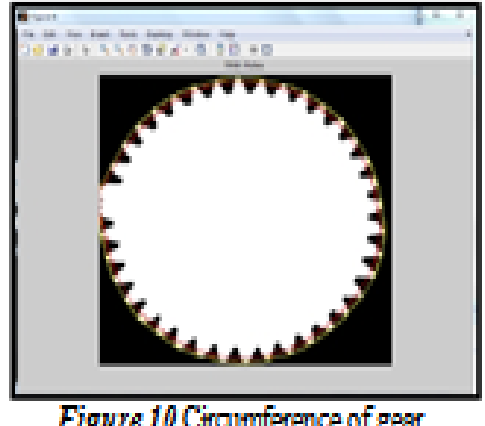

The above image shows the step of a teeth calculating algorithm where it identify where it identify the outer as well as inner circumference of teeth based on Teeth counting algorithm.

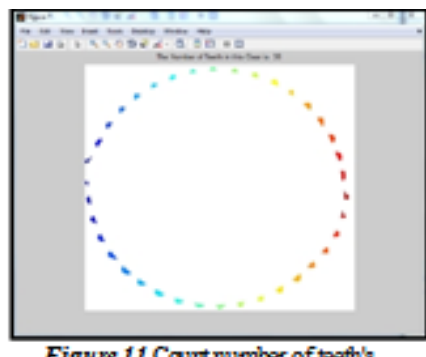

Figure 11 Count number of teeth's

The above image count the number of teeth's of the gear image for checking whether the number of teeth's are same as that of the subscribe number. If the number of teeth matches with the subscribe number then the gear in non- defective otherwise it is defected. 


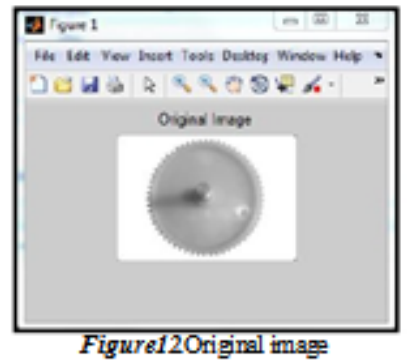

This figure shows an RGB image of the original plastic gear which is used to make industrial products like robotics.

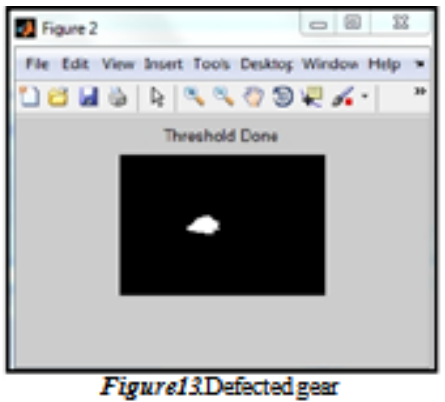

The above image basically shows the location of the defect after running the defect identifier algorithm based on thresholding.

\section{Conclusion and Future Scope}

Firstly, in this paper we have been able to detect defective gears which have specific number of teeths.If the number of teeth's are deformed or more or less than the subscribe number than the gear is defective in nature and therefore the gear is useless. Secondly, we have been able to identify some surface defects by using range bound thresholding which can work on both color and grey scale images of the gears. After conducting the above procedure for defect detection we suggest that in future we must take advantage of some machine learning algorithms for making defects detecting more reliable and robust. There are number of future possibilities for improving the performance of these detection algorithms like usage of machine algorithms which help to identify the defective parts as these occur over a period of time. They increase their accuracy based on the updated parameter set and scenario machine algorithm like Support Vector Machine, K-NN and neural network can be used.

\section{Acknowledgment}

I would like to thanks my parents and my friends for their support and trust.

\section{References}

[1] Amandeep Mavi, Mandeep Kaur,(2012) - Identify defects in Plastic (gears) using Digital image processing -A Review IRACST - International Journal of Computer Science and Information Technology \& Security (IJCSITS), ISSN: $2249-9555$ Vol. 2, No.2, April 2012

[2] Alisha Tremaine (2005), — Characterization of Internal Defects in Open Die Forgings\| FIERF Grant Project for Undergraduate Research November 17, 2005

[3] S. Kamaruddin, Zahid A. Khan and S. H. Foong(2010) - Application of Taguchi Method in the Optimization of Injection Moulding Parameters for Manufacturing Products from Plastic Blend \| IACSIT International Journal of Engineering and Technology, Vol.2, No.6, December 2010 ISSN: 1793-8236

[4] B. Mrazovac, M. Z. Bjelica, I. Papp, N. Teslic, "Smart Audio/Video Playback Control Based On Presence Detection And User Localization In Home Environment", In Proceedings Of Ecbs-Eerc 2011, Full-Paper, Bratislava, Slovakia 2011, Pp. 44-53

[5] www.enggpapers.com. 


\section{AUTHORS PROFILE}

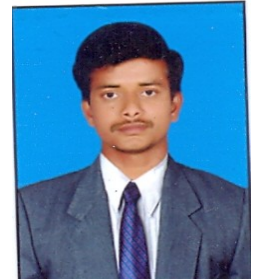

Mr. A.Dasthagiraiah-He is Masters of Technology in Electronics and communication Engineering from Hindustan University in the year 2011 with specialization in Embedded Systems. He has given guidance to many students in their thesis work of M.Tech. He has also contributed in the research work on Digital Image Processing with his papers. He has four years teaching Experience and presently working as Asst. Professor in Mekapati Rajamohan Reddy Institute of Technology and Science ,Udayagiri,SPSR Nellore. He has done Bachelor's of Technology from JNTUA University in the year 2009 in Electronics and communication Engineering.

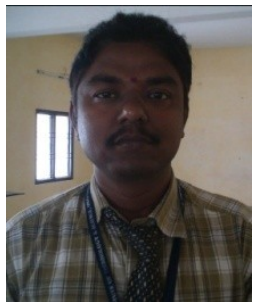

Mr. N.V.Subba Reddy-He is Masters of Technology in Electronics and communication Engineering from JNTUA University in the year 2010 with specialization in VLSI Design. He has given guidance to many students in their thesis work of M.Tech. He has also contributed in the research work on Digital Image Processing with his papers. He has Nine years teaching Experience and presently working as Assoc. Professor in Mekapati Rajamohan Reddy Institute of Technology and Science ,Udayagiri,SPSR Nellore. He has done Bachelor's of Technology from JNTUA University in the year 2003 in Electronics and communication Engineering. 